healthcare complaint, yet information-sharing following stroke is little-researched.

Aim To describe and explore: a) carers' experiences of professional truth-telling in the 6 months following severe stroke b) how carers process uncertainty

Methods Secondary analysis of semi-structured serial interviews with carers at 6 weeks and 6 months post-stroke, using Framework approach.

Population: Informal carers of severely-dependent adult patients with Total Anterior Circulation Stroke in Lothian (Scotland), including carers of patients who died during the study.

Sampling: Purposive sampling for maximal variation; secondary analysis sampled geographically.

Rigor: Analysis of deductive and inductive themes; discussion of emergent themes with primary data collectors; examination of deviant, negative and extreme cases.

Results 20 transcripts from 15 carers were analysed, including 6 bereavement interviews. All interviews referenced truth-telling by healthcare professionals, most frequently 'construction of truth as a process' and 'value of honesty/frankness'. Some carers described professionals intending to deceive. Most carers described uncertainty as something negative, using behaviours to reduce uncertainty. A minority described uncertainty as a positive or 'window for hope', using behaviours to increase uncertainty.

Carer, professional, patient and environmental factors influenced carers' experiences. What carers said was complex and views changed over time.

Discussion and conclusions We present a new visual model of truth-telling between healthcare professionals and carers. Truth-telling is a complex, two-way process. Carers and professionals view 'the truth' through 'lenses' of their own worldviews, experiences and knowledge. Carers' views of uncertainty affect engagement with truth-telling. The truth-telling system is open, asymmetrical and dynamic: with access of external information and a gradient of knowledge and power within each professional-carer dyad, which changes over time. Poor communication has important fall-out. Our visual model could help professionals approach information-sharing following severe stroke more confidently.

\section{DEVELOPMENT OF A RESOURCE FOR FAMILY CARERS OF PEOPLE LIVING WITH DEMENTIA WITH NUTRITION AND HYDRATION DIFFICULTIES TOWARDS THE END-OF- LIFE}

\begin{abstract}
${ }^{1}$ Yolanda Barrado-Martín, ${ }^{1}$ Pushpa Nair, ${ }^{2,3}$ Kanthee Anantapong, 2,4 Kirsten J Moore, ${ }^{5}$ Christina H Smith, ${ }^{1}$ Greta Rait, ${ }^{2,6}$ Elizabeth L Sampson, ${ }^{7}$ Jill Manthorpe, ${ }^{1,2}$ Nathan Davies. ${ }^{1}$ Research Department of Primary Care and Population Health, UCL; ${ }^{2}$ Marie Curie Palliative Care Research Department, UCL; ${ }^{3}$ Department of Psychiatry, Prince of Songkla University; ${ }^{4}$ National Ageing Research Institute, Parkville, Victoria, Australia; ${ }^{5}$ Language and Cognition, Division of Psychology and Language Sciences, UCL; ${ }^{6}$ Barnet Enfield and Haringey Mental Health Trust Liaison Team, North Middlesex University Hospital; ${ }^{7}$ NIHR Policy Research Unit in Health and Social Care Workforce Research Unit, and NIHR Applied Research Collaborative (ARC) South London, King's College London
\end{abstract}

\subsection{6/spcare-2021-PCC.14}

Background Family carers often struggle to support relatives living with dementia with nutrition and hydration towards the end-of-life. The aim of this study was to co-design with family carers and professionals an information resource to support family carers with this task.
Methods This study consisted of four phases: 1) qualitative systematic review; 2) a scoping review of existing resources; 3) 61 qualitative interviews with people with dementia, professionals, and family carers; 4) data synthesis and co-design development of an information resource.

We constructed a matrix synthesising the key findings from phases $1-3$, devising themes of the key content across the evidence and providing summaries from each source of evidence. The matrix provided a transparent pathway of the evidence and content for the design process.

For the co-design phase 18 participants were recruited, 9 family carers and 9 professionals, split into two mixed groups. Four co-design workshops were conducted (two with each group) to develop the resource. During the workshops participants were presented with a summary of results from the matrix. Using modified nominal group processes and a series of tasks including categorisation and ranking we produced an initial prototype of the resource. The prototype was refined in subsequent co-design meetings and via email with individual participants.

Results The main areas covered within the resource include: eating and drinking difficulties in dementia; end of life; managing swallowing difficulties; points to discuss with professionals in future appointments; support for family carers and; further resources. Information is presented in a booklet with a mix of formats including: diagrams; quotes; top tips; flow charts; and visual images.

Conclusions This practical resource is the first to focus on nutrition and hydration towards the end-of-life in dementia care. This study provides a transparent stepwise approach to developing interventions, key for future similar studies.

\section{DEATHBED ETIQUETTE-SUPPORT FOR BEING AT THE BEDSIDE OF THE PERSON YOU LOVE}

Amy Gadoud, Karen Sanders, Amarachukwu Nwosu, Joanne Elverson, Lynn Bassett, Margaret Doherty. Lancaster Medical School and Trinity Hospice, Blackpool; Institute of Business, Law and Society, St Mary's University, Twickenham; Lancaster Medical School and Royal Liverpool Hospital; St Oswald's Hospice, Newcastle; Art of Dying Well, St Mary's University, Twickenham

\subsection{6/spcare-2021-PCC.15}

Background People in the UK are often uncomfortable with supporting the dying. As the population ages we are less likely to have supported a loved one who has died.

Methods The Centre for the Art of Dying Well, with palliative care experts and experts by experience, created a resource to support someone at the deathbed of a loved one. This was updated for the COVID-19 pandemic. This abstract describes the wider public engagement with this resource, using content analysis to evaluate public sentiment and understanding of it.

Results Release of the original Guide coincided with a report assessing preparedness for supporting someone who is dying, a podcast and a press release. It was widely quoted in the news media with reports in The Times; local and national news (Radio Oxfordshire, Talk Radio, Channel 5 News). Google Analytics demonstrated 7,341 unique visitors to the Guide spending, on average, 2 minutes 2 seconds reading it. The version updated for COVID-19 was widely cited in the Financial Times, the Daily Mail, the Sun and Vatican News and an interview on 5 Live Radio. Based on an analysis of article comments, public sentiment and understanding were shown to 